\title{
A New Combined Raman and Polarization Holographic Approach for Sensing Circulating Tumor Cells ${ }^{+}$
}

\author{
Maria Mangini *, Antonella Ferrara, Gianluigi Zito, Stefano Managò, Alberto Luini, Giuseppe \\ Coppola and Anna Chiara De Luca \\ * Correspondence: maria.mangini@ibbc.cnr.it \\ + Presented at the 7th International Electronic Conference on Sensors and Applications, 15-30 November \\ 2020; Available online: https://ecsa-7.sciforum.net/.
}

Published: 15 November 2020

\begin{abstract}
Circulating tumor cells (CTCs) are a rare subgroup of cells that detach from the primary solid tumor and circulate in the bloodstream of cancer patients. These cells act as a seed for metastases; moreover, they maintain the primary tumor heterogeneity and mimic tumor properties. All these features make CTCs good candidates to be used as clinical biomarker for patient diagnosis, prognosis and treatment. Indeed, the isolation and characterization of CTCs can smooth the way to apply precision medicine approach in cancer patients. However, the current methods to detect CTCs are mainly based on the recognition of epithelial cell adhesion molecule (EpCAM) expressed on cancer cell membrane, but they typically fail to detect tumoral cells that are losing epithelial phenotype and starting invasion/metastatic process. Here, we used deuterium as vibrational tag to develop a new CTCs detection method based on Raman spectroscopy potentially able to detect any type of CTCs. In particular, we exploited the capacity of cancer cell to internalize and metabolize glucose 5-10 faster than normal cell, the so-called Warburg effect. Normal prostatic cells (PNT2), cancer prostatic (PC3) and hepatic (HepG2) cells were used as in vitro model. Cells were cultured in presence of $25 \mathrm{mM}$ deuterated glucose for $48 \mathrm{~h}$ and then analyzed by Raman spectroscopy (1). The typical deuterium Raman band at $2100 \mathrm{~cm}^{-1}$ was present in the spectra of PC3 and HepG2 cells, but not in PNT2 spectra. These results indicate the presence of Warburg effect in our cellular model and that cancer cells can be differentiated from normal cells following glucose metabolism. To simulate the presence of CTCs in blood, PC3 and HepG2 cells were co-cultured with white blood cells isolated from healthy donor blood in presence of deuterated glucose. The deuterium Raman signal was observable only in the spectra of PC 3 and HepG2 cells. Our data demonstrate that cancer cells can be distinguished from healthy cells independently from EpCAM expression just exploiting the glycolytic metabolism also when they are in the same media. These results shed a light on the possibility to develop new CTCs detecting methods using label-free approach based on Raman spectroscopy.
\end{abstract}

\section{References}

1. Managò, S.; Valente, C.; Mirabelli, P.; Circolo, D.; Basile, F.; Corda, D.; De Luca, A.C. A reliable Ramanspectroscopy-based approach for diagnosis, classification and follow-up of B-cell acute lymphoblastic leukemia. Sci. Rep. 2016, 6, 24821. 